\title{
On the Empirical Estimation of Utility Distribution Damping Parameters Using Power Quality Waveform Data
}

\author{
Kyeon Hur, ${ }^{1}$ Surya Santoso, ${ }^{1}$ and Irene Y. H. Gu ${ }^{2}$ \\ ${ }^{1}$ Department of Electrical and Computer Engineering, The University of Texas at Austin, Austin, TX 78712, USA \\ ${ }^{2}$ Department of Signals and Systems, Chalmers University of Technology, 41296 Gothenburg, Sweden
}

Received 30 April 2006; Revised 18 December 2006; Accepted 24 December 2006

Recommended by M. Reza Iravani

\begin{abstract}
This paper describes an efficient yet accurate methodology for estimating system damping. The proposed technique is based on linear dynamic system theory and the Hilbert damping analysis. The proposed technique requires capacitor switching waveforms only. The detected envelope of the intrinsic transient portion of the voltage waveform after capacitor bank energizing and its decay rate along with the damped resonant frequency are used to quantify effective $X / R$ ratio of a system. Thus, the proposed method provides complete knowledge of system impedance characteristics. The estimated system damping can also be used to evaluate the system vulnerability to various PQ disturbances, particularly resonance phenomena, so that a utility may take preventive measures and improve PQ of the system.
\end{abstract}

Copyright @ 2007 Kyeon Hur et al. This is an open access article distributed under the Creative Commons Attribution License, which permits unrestricted use, distribution, and reproduction in any medium, provided the original work is properly cited.

\section{INTRODUCTION}

Harmonic resonance in a utility distribution system can occur when the system natural resonant frequency-formed by the overall system inductance and the capacitance of a capacitor bank-is excited by relatively small harmonic currents from nonlinear loads [1]. The system voltage and current may be amplified and highly distorted during the resonance encounter. This scenario is more likely to occur when a capacitor bank is energized in a weak system with little or negligible resistive damping. During a resonance, the voltage drop across the substation transformer and current flowing in the capacitor bank is magnified by $Q$ times. $Q$ is the quality factor of a resonant circuit and is generally represented by $X_{L} / R$, where $X_{L}$ and $R$ are the reactance and resistance of the distribution system Thevenin equivalent source and substation transformer at the resonant frequency. Note that during a resonance, the magnitude of $X_{L}$ is equal to but opposite in sign to that of $X_{C}$, the reactance of a capacitor bank. In addition, during a resonance, $X_{L}$ and $X_{C}$ reactances are $h$ and $1 / h$ multiple of their respective fundamental frequency reactance, where $h$ is the harmonic order of the resonant frequency. Due to the highly distorted voltage and current, the impacts of harmonic resonance can be wide ranging, from louder noise to overheating and failure of capacitors and transformers $[1,2]$.
Based on this background, it is desirable to predict the likelihood of harmonic resonance using system damping parameters such as the $Q$ factor and the damping ratio $\zeta$ at the resonance frequency. The $Q$ factor is more commonly known as the $X / R$ ratio. The reactance and resistance forming the $Q$ factor should be the impedance effective values that include the effect of loads and feeder lines, in addition to impedances from the equivalent Thevenin source and substation transformer. In other words, the $X / R$ ratio is influenced by the load level. When the ratio is high, harmonic resonance is more likely to occur. Therefore, this paper proposes an effective algorithm to estimate the $X / R$ ratio based on linear dynamic system theory and the Hilbert damping analysis. The estimation requires only voltage waveforms from the energization of capacitor banks to determine the overall system damping. It does not require system data and topology, and therefore it is practical to deploy in an actual distribution system environment.

There has been very little research carried out on this subject. Most previous efforts have been exerted on voltage stability issues in the transmission system level, such as dynamic load modeling, and its impacts on intermachine oscillations and designing damping controllers [3-5]. Very little research has been conducted to quantify the damping level of the power system, particularly distribution feeders. Research has shown that the system damping supplied by resistive 


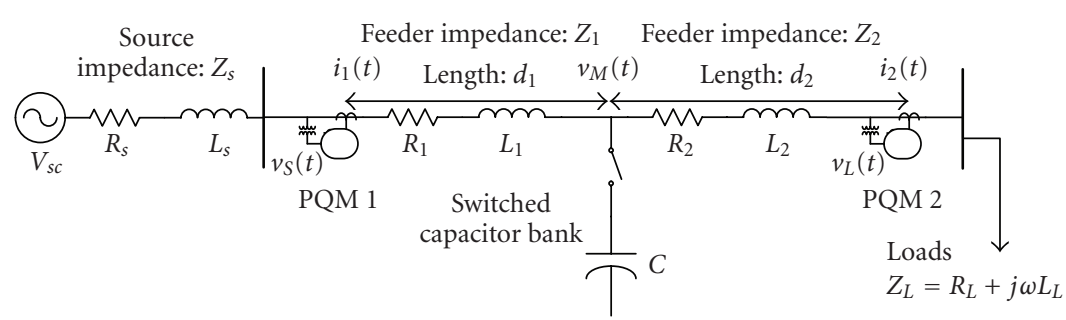

FIgURE 1: One-line diagram for a typical utility distribution feeder.

components of the feeder lines and loads have a beneficial impact in preventing catastrophic resonance phenomena $[1,2]$. However, a few other studies on the application of signal processing techniques to harmonic studies have been undertaken on the assumption that harmonic components are exponentially damped sinusoids. Those techniques include ESPRIT [6], Prony analysis [4], and system identification based on the all-pole (AR) model [7]. These techniques can help better explain the characteristics of individual harmonic components. Those techniques need to clear some significant issues such as intrinsic spurious harmonics that may mislead the evaluation of the results, the uncertainty of the system order and the computational burden that prevent real-world applications. Unfortunately, no work has been extended to quantify the overall damping of the system.

The organization of this paper is as follows. Section 2 describes the scope of the problem and develops a smart algorithm for estimating power system damping using capacitor switching transient data based on Hilbert transform and linear dynamic system theory in Section 3. Section 4 demonstrates the efficiency of the proposed technique using data from an IEEE Test Feeder [8] modeled in the time-domain power system simulator [9] and actual measurement data in Section 5. The paper concludes in Section 7.

\section{PROBLEM DESCRIPTION AND SCOPE OF THE PROBLEM}

Let us consider a one-line diagram for a power distribution system in Figure 1, where a shunt capacitor bank is installed in the distribution feeder and power quality monitoring devices are located on both sides of the capacitor bank. When the capacitor bank is energized, an oscillatory transient can be observed in the voltage and current waveforms captured by the power quality monitors. The oscillation frequency is indeed the new natural power system resonant frequency formed by the equivalent inductance and the capacitance of the switched capacitor bank.

The problem addressed in this paper can be stated as follows: given voltage waveforms as a result of capacitor energizing, determine the effective $X / R$ ratio for the resonant frequency at the particular bus of interest. The proposed method makes use of the transient portion of capacitor switching waveforms captured anywhere in the system. Thus, the proposed method works well only with capacitors en- ergized without any mechanism to reduce overvoltage transients. Therefore, the capacitor banks considered in this work are those energized with mechanical oil switches. This is representative of the banks found in the majority of distribution feeders.

\section{POWER SYSTEM DAMPING ESTIMATION}

The estimation of the system damping quantified in terms of the $X / R$ ratio and the damping ratio $\zeta$ requires the use of the Hilbert transform and the theoretical analysis of the distribution circuit. The Hilbert transform is used to determine and extract the circuit properties embedded in the envelope of the waveshape of the capacitor switching transient waveform. A brief review of the transform is described in Section 3.1. The circuit analysis derives and shows the envelope of the transient waveform which contains the signature of the $X / R$ ratio. Section 3.2 analyzes the derivation and analysis in detail and discusses practical consideration.

\subsection{Hilbert transform}

The Hilbert transform of a real-valued time domain signal $y(t)$ is another real-valued time domain signal, $\tilde{y}(t)$, such that an analytic signal $z(t)=y(t)+j \tilde{y}(t)$ exists [10]. This is a generalization of Euler's formula in the form of the complex analytic signal. It is also defined as a 90-degree phase shift system as shown below:

$$
\begin{gathered}
\tilde{y}(t)=H[y(t)]=\int_{-\infty}^{\infty} \frac{y(\tau)}{\pi(t-\tau)} d \tau=y(t) * \frac{1}{\pi t}, \\
F[\tilde{y}(t)]=\tilde{Y}(f)=(-j \operatorname{sgn} f) Y(f),
\end{gathered}
$$

where $\tilde{Y}(f)$ is the Fourier transform of $\tilde{y}(t)$. From $z(t)$, we can also write $z(t)=a(t) \cdot e^{j \theta(t)}$, where $a(t)$ is the envelope signal of $y(t)$, and $\theta(t)$ is the instantaneous phase signal of $y(t)$. The envelope signal is given by $a(t)=\sqrt{y(t)^{2}+\tilde{y}(t)^{2}}$ and the instantaneous phase, $\theta(t)=\tan ^{-1}(\tilde{y}(t) / y(t))$. Using the property in the second equation of (1), one can easily obtain the Hilbert transform of a signal, $y(t)$. Let $Z(f)$ be the Fourier transform of $z(t)$ and one can obtain the following 
relations:

$$
\begin{aligned}
Z(f) & =F[z(t)]=F[y(t)+j \tilde{y}(t)] \\
& =Y(f)+j \tilde{Y}(f) \\
& =(1+\operatorname{sgn} f) Y(f) \\
& = \begin{cases}2 Y(f) & \text { for } f>0, \\
0 & \text { for } f<0,\end{cases} \\
z(t) & =F^{-1}[Z(f)]=y(t)+j \tilde{y}(t) .
\end{aligned}
$$

Thus, the inverse Fourier transform of $Z(f)$ gives $z(t)$ as shown in (3). For the case of quadratic damping, the decaying transient and its Hilbert transform can be represented as

$$
\begin{aligned}
& y(t)=y_{m} e^{-\zeta \omega_{n} t} \cos \left(\omega_{d} t+\phi\right), \\
& \tilde{y}(t)=y_{m} e^{-\zeta \omega_{n} t} \sin \left(\omega_{d} t+\phi\right) .
\end{aligned}
$$

Thus, the resulting envelope, $a(t)$, becomes $y_{m} e^{-\zeta \omega_{n} t}$, where $y_{m}$ is an arbitrary constant magnitude. This is a unique property of the Hilbert transform applicable to envelope detection.

\subsection{Algorithm development}

\subsubsection{Analysis of the distribution system and definition of the effective $X / R$ ratio}

Let us assume that the distribution system is balanced. Therefore, the Thevenin equivalent source impedance is represented with $R_{s}$ and $L_{s}$, while the line impedance for segments $d_{1}$ and $d_{2}$ are represented with its positive sequence impedance $\left(r+j \omega L_{u}\right) d_{1}=R_{1}+j \omega L_{1}$ and $\left(r+j \omega L_{u}\right) d_{2}=$ $R_{2}+j \omega L_{2}$, where $r$ and $L_{u}$ are the line resistance and inductance in per unit length. The load impedance is represented with $Z_{L}=R_{L}+j X_{L}$. Let voltage $v_{s}(t), i_{1}(t)$ and $v_{L}(t), i_{2}(t)$ be the instantaneous voltages and currents measured by PQM 1 and PQM 2, respectively, and let $v_{M}(t)$ be the voltage over the capacitor bank.

Thus, one can set up the following differential equations for the equivalent circuit immediately following the energization of the capacitor bank, that is, $t=0^{+}$. Note that currents $i_{1}$ and $i_{2}$ are measured by PQM 1 and 2 in the direction of the prevailing system loads as denoted in Figure 1. In the vectormatrix form, the state equations and observation equations are expressed as

$$
\begin{gathered}
\dot{\mathbf{x}}(t)=\mathbf{A x}(t)+\mathbf{B u}(t), \\
\mathbf{y}(t)=\mathbf{C x}(t),
\end{gathered}
$$

where

$$
\mathbf{x}(t)=\left[\begin{array}{lll}
\frac{d i_{1}}{d t} & \frac{d i_{2}}{d t} & \frac{d v_{M}}{d t}
\end{array}\right]^{T},
$$

$$
\begin{gathered}
\mathbf{A}=\left[\begin{array}{ccc}
\frac{-\left(R_{s}+R_{1}\right)}{L_{s}+L_{1}} & 0 & \frac{-1}{L_{s}+L_{1}} \\
0 & \frac{-\left(R_{2}+R_{L}\right)}{L_{2}+L_{L}} & \frac{1}{L_{2}+L_{L}} \\
\frac{1}{C} & \frac{-1}{C} & 0
\end{array}\right], \\
\mathbf{B}=\left[\begin{array}{l}
1 \\
0 \\
0
\end{array}\right], \quad \mathbf{C}=\mathbf{I}_{3},
\end{gathered}
$$

and $\mathbf{y}(t)$ is the output vector, $\mathbf{x}(t)$ the state vector, and $\mathbf{u}(t)$ the input vector. The input vector, $\mathbf{u}(t)$, of this system comprises only the equivalent voltage source. The state vector is regarded as the output vector. Thus, matrix $\mathbf{C}$ is a $3 \times 3$ identity matrix. Let the transfer function $G(s)$, which describes the behavior between the input and output vectors, be expressed in the following form [11]:

$$
G(s)=\mathbf{C}(s \mathbf{I}-\mathbf{A})^{-1} \mathbf{B}=\left[G_{1}(s), G_{2}(s), G_{3}(s)\right]^{T},
$$

where

$$
\begin{gathered}
G_{1}(s)=\frac{C\left(L_{2}+L_{L}\right) s^{2}+\left(R_{L}+R_{2}\right) C s+1}{\Delta}, \\
G_{2}(s)=\frac{1}{\Delta}, \\
G_{3}(s)=\frac{\left(L_{2}+L_{L}\right) s+\left(R_{2}+R_{L}\right)}{\Delta},
\end{gathered}
$$

and $\Delta$ is a characteristic equation of the system and is represented as follows:

$$
\begin{aligned}
\Delta= & |s \mathbf{I}-\mathbf{A}| \\
= & \left(L_{s}+L_{1}\right)\left(L_{2}+L_{L}\right) C s^{3} \\
& +\left\{\left(L_{s}+L_{1}\right)\left(R_{2}+R_{L}\right)+\left(L_{2}+L_{L}\right)\left(R_{s}+R_{1}\right)\right\} C s^{2} \\
& +\left\{\left(R_{s}+R_{1}\right)\left(R_{2}+R_{L}\right) C+\left(L_{s}+L_{1}+L_{2}+L_{L}\right)\right\} s \\
& +R_{s}+R_{1}+R_{2}+R_{L} .
\end{aligned}
$$

The $s$-domain representation of voltages at PQM $1\left(V_{S}(s)\right)$, PQM $2\left(V_{L}(s)\right)$ and across capacitor $\left(V_{M}(s)\right)$ can be obtained as follows:

$$
\begin{gathered}
V_{S}(s)=V_{s c}(s)\left\{G_{1}(s)\left(R_{1}+s L_{1}\right)+G_{3}(s)\right\} \\
V_{L}(s)=V_{s c}(s) G_{2}(s)\left(L_{L} s+R_{L}\right) \\
V_{M}(s)=V_{s c}(s) G_{3}(s)
\end{gathered}
$$

Since the power system fundamental frequency is substantially lower than a typical capacitor switching frequency [12], the input source voltage is considered constant in $s$ domain,

$$
V_{s c}(s)=\frac{v_{s c}\left(t_{s}^{-}\right)}{s},
$$


where $v_{s c}\left(t_{s}^{-}\right)$indicates a voltage level immediately before switching. Note that the roots of the characteristic equation are the eigenvalues of the matrix $\mathbf{A}$, and the order of the characteristic equation is three. In linear dynamic system theory, the characteristic equation of the second-order prototype system is generally considered, that is,

$$
\Delta(s)=s^{2}+2 \zeta \omega_{n} s+\omega_{n}^{2},
$$

where $\omega_{n}$ and $\zeta$ are the resonant frequency and the system damping ratio, respectively. The series $R L C$ circuit is one of the representative second-order prototype systems, which is the case of an isolated capacitor bank. Neglecting the circuit downstream from the capacitor bank, one can obtain the following characteristic equation:

$$
\Delta(s)=s^{2}+\left(\frac{R_{s}+R_{1}}{L_{s}+L_{1}}\right) s+\frac{1}{\left(L_{s}+L_{1}\right) C} .
$$

Thus, we obtain the following relations:

$$
\omega_{n}^{2}=\frac{1}{\left(L_{s}+L_{1}\right) C}, \quad 2 \zeta \omega_{n}=\frac{R_{s}+R_{1}}{L_{s}+L_{1}} .
$$

From (14), we obtain the damping ratio of the system:

$$
\zeta=\frac{1}{2 \omega_{n}}\left(\frac{R_{s}+R_{1}}{L_{s}+L_{1}}\right)
$$

In fact, (15) derives the conventional $X / R$ ratio of the system at the resonant frequency, which frequently appears in power system literature to describe the system resonance, that is, the so-called quality factor, $Q$,

$$
\frac{X}{R}=\frac{1}{2 \zeta}=\omega_{n}\left(\frac{L_{s}+L_{1}}{R_{s}+R_{1}}\right)
$$

Note that the behavior of the transient voltage measured in the utility system after energizing the capacitor bank can be described by the general exponential function in the same form as (4). Hence, transient voltage can be described as follows:

$$
\begin{aligned}
v(t) & =v(0) e^{-\zeta \omega_{n} t}\left(p \cos \left(\omega_{d} t\right)+q \sin \left(\omega_{d} t\right)\right) \\
& =r e^{-\zeta \omega_{n} t} \cos \left(\omega_{d} t+\phi\right) \\
& =a(t) \cos \left(\omega_{d} t+\phi\right),
\end{aligned}
$$

where $v(0)$ is an initial condition, $\omega_{d}=\omega_{n} \sqrt{\left(1-\zeta^{2}\right)}$ is the damped resonant frequency, $p$ and $q$ are arbitrary constants, $r=\sqrt{p^{2}+q^{2}}$ and $\phi=-\tan ^{-1}(q / p)$. Keep in mind that the aforementioned equations are based on the series $R L C$ circuit without considering loads. Thus, the $X / R$ ratio does not include damping contributions of the loads and the downstream lines to the whole system.

We should emphasize that the damping ratio is not strictly defined in the higher order system. However, thorough numerical analyses prove that the characteristic equation in (9) can be reasonably represented by a pair of complex conjugate dominant poles and one insignificant pole that is further away from $j \omega$ axis in the left half $s$-domain than those of dominant poles. Therefore, its effect on transient response is negligible, which corresponds to the fast-decaying time response. Application of the model reduction method [13] to the voltages of interest also confirms that the transfer functions of $V_{s}(s)$ and $V_{L}(s)$ in (10) can be reduced, and the transfer function of $V_{M}(s)$ in (10) can be approximated after truncating the fast mode as follows:

$$
\begin{gathered}
V_{S}(s) \approx V_{s c}(s)\left(Q \frac{s^{2}+2 \zeta_{1} \omega_{n 1} s+\omega_{n 1}^{2}}{s^{2}+2 \zeta_{2} \omega_{n 2} s+\omega_{n 2}^{2}}\right), \\
V_{M}(s) \approx V_{s c}(s)\left(\frac{-K s+P}{s^{2}+2 \zeta \omega_{n} s+\omega_{n}^{2}}\right),
\end{gathered}
$$

where $Q, K$, and $P$ are arbitrary constants. $V_{L}(s)$ can be reduced to the same form as $V_{S}(s)$. Note that the damping term of the reduced second-order system is a function of line parameters and loads. Thus, it should not be interpreted as the conventional $X / R$ ratio which is a function only of upstream lines and source parameters as defined in (16). However, the approximate damping term can indicate the relative $X / R$ ratio of the whole system effectively and can quantify the overall contributions to the system damping by both lines and loads. Thus, the paper defines $1 /(2 \zeta)$ from the reduced second-order characteristic equation as the effective $X / R$ ratio of the system. What is worthnoting is that the characteristic equation can vary according to the load composition. Hence, the $X / R$ ratio is not a unique function of the parameters of the lines and loads but depends on the load composition and line configuration. Note that a parallel representation of the load elements results in a fourth-order characteristic equation. However, the fourth-order system can also be reduced to the second-order prototype system by the model reduction technique with much bigger damping ratio than that from the series load representation even under the same loading condition. This is briefly illustrated in Section 4, but the details are beyond the scope of the paper. Therefore, the transient response of the whole system can be described by (17) as well. This is the motivation for detecting the envelope of the transient voltage by means of Hilbert transform. Consequently, the exponent, $-\zeta \omega_{n}$, of (17) can lead to the effective $X / R$ ratio or $1 /(2 \zeta)$ if $\omega_{n}$ is available. Since the aforementioned system parameters for determining the system damping level are not readily available, we propose an empirical method using conventional PQ data for evaluating the effective $X / R$ ratio. The following section discusses how to obtain the effective $X / R$ ratio of the system using conventional capacitor switching transient data.

\subsubsection{Implementation and practical consideration}

The implementation of the proposed damping estimation technique is illustrated in Figure 2. The implementation begins with an existing PQ database or a real-time PQ data stream as used in web-based monitoring devices. Since typical PQ monitors capture a wide range of disturbance events, a separate algorithm is needed to distinguish capacitor switching event data from other PQ data. The identification of capacitor switching transient waveforms can be done visually 


\begin{tabular}{|c|c|c|c|c|}
\hline $\begin{array}{c}\text { PQ data and } \\
\text { system } \\
\text { information }\end{array}$ & \multicolumn{2}{|c|}{$\begin{array}{l}\text { Capacitor switching } \\
\text { identification }\end{array}$} & \multirow{2}{*}{$\begin{array}{l}\text { - Switching instant } \\
\text { - Number of samples } \\
\text { - Sampling rate }\end{array}$} & $\begin{array}{l}\text { Empirical identification of the } \\
\text { free response of the capacitor } \\
\text { bank energizing }\end{array}$ \\
\hline & & & & $\downarrow$ \\
\hline \multirow{2}{*}{\multicolumn{2}{|c|}{$\begin{array}{l}\text { Quantification of the } \\
\text { system damping } \\
\text { - Compute the damping ratio using the } \\
\text { relationship between the slope and the } \\
\text { resonant frequency } \\
\text { - Quantify the system damping }(X / R \\
\text { ratio) based on the second-order } \\
\text { prototype system }\end{array}$}} & \multirow{2}{*}{\multicolumn{2}{|c|}{$\begin{array}{l}\text { Hilbert transform analysis } \\
\text { - Apply the Hilbert transform } \\
\text { analysis to the free response signal } \\
\text { - Obtain the envelope data, } a(t) \text {, } \\
\text { and its logarithm } \\
\text { - Perform linear regression and } \\
\text { estimate the slope parameter, } \zeta \omega_{n}\end{array}$}} & \begin{tabular}{l}
\multicolumn{1}{c}{ Spectral analysis } \\
- Perform FFT on the \\
free response signal and \\
obtain dominant system \\
resonant frequency
\end{tabular} \\
\hline & & & & Hilbert damping analysis \\
\hline
\end{tabular}

FIgURE 2: Data flow and process diagram of the system damping estimation.

or automatically $[7,14]$. Once a single event of capacitor switching transient data, that is, three-phase voltage, is identified, we extract transient portions of voltage waveforms after switching and construct extrapolated voltage waveforms based on the steady state waveforms after capacitor energizing. This extrapolation can be done by concatenating a single period of waveforms captured 3 or 4 cycles after the switching operation on the assumption that voltage signals are considered to be (quasi-)stationary for that short period of time. If the number of samples after the detected switching instant is not sufficient to form a single period, steady-state voltage data before capacitor switching can be used alternatively. It is not uncommon to observe this situation since most of the PQ monitors store six cycles of data based on the uncertain triggering instant. Wavelet transform techniques, among others, are most frequently used for effectively determining the exact switching instant [14]. For example, there exists a commercial power quality monitoring system equipped with singularity (switching) detection based on the wavelet transform.

In this effort, we assume that switching time instant can be accurately detected. Then, we subtract the second from the first and get the differential portions that are free from the harmonics already inherent in the system and the voltage rise due to reactive energy compensation. This differential portion can be interpreted as the zero-input (free) response of the system, whose behavior is dictated by the characteristic equation as discussed in Section 3.2.1. The process of deriving this empirical-free response of the capacitor bank energizing is more detailed in [15]. The Hilbert transform is then performed to find the envelope signal, $a(t)$, of (17). In fact, the envelope from the Hilbert transform is not an ideal exponential function and is full of transients especially for those low-magnitude portions of the signal approaching the steady-state value (ideally zero). Thus, only a small number of data are utilized in order to depict the exponential satisfactorily: one cycle of data from the capacitor switching instant is generally sufficient to produce a good exponential shape. The number of data will depend on the sampling rate of the PQ monitoring devices and should be calibrated by investigating the general load condition, especially when the method is applied to a new power system in order to optimize the performance. The obtained data is now fitted into an exponential function. The direct way to fit the data into the exponential function is possible through iteration-based nonlinear optimization technique. However, the exponential function is namely an intrinsic linear function, such that the $\ln a(t)$ produces a linear function, that is,

$$
\ln \{a(t)\}=\ln r-\zeta \omega_{n} t
$$

As a result, we can apply standard least squares method to approximate the optimal parameters more efficiently [16]. The solution is not optimal in minimizing the squared error measure, due to the logarithmic transformation. However, except for very high damping cases, this transformation plus the least squares estimation method, creates a very accurate estimate of $a(t)$. The FFTs of the differential voltages may also provide good spectral information of the system since the FFTs are performed on the data virtually free from inherent harmonic components that may produce spurious resonant frequency components [15]. Thus, one can obtain the effective $X / R$ ratio that quantifies the system damping level, including impacts from lines and loads. The proposed algorithm is very practical and ready to be implemented in modern PQ monitoring systems since the conventional capacitor switching transient data is all it needs and the method is not computationally intensive.

\section{METHOD VALIDATION USING IEEE TEST MODEL}

This section demonstrates the application of the damping estimation method using the IEEE power distribution test feeder [8]. The test system is a $12.47 \mathrm{kV}$ radial distribution system served by a $12 \mathrm{MVA} 115 / 12.47 \mathrm{kV}$ delta-Yg transformer. The Thevenin equivalent impedance is largely due to the transformer leakage impedance, that is, $Z(\%)=(1+j 10)$ on a 12 MVA base. Thus, the equivalent source inductance $L_{s}$ would be $3.4372 \mathrm{mH}$. The evaluation of distance estimates is carried out under both unbalanced $\left[Z_{012}\right]_{U B}(\Omega / m i)$ and balanced $\left[Z_{012}\right]_{B}(\Omega / m i)$. Their sequence impedance matrices in 


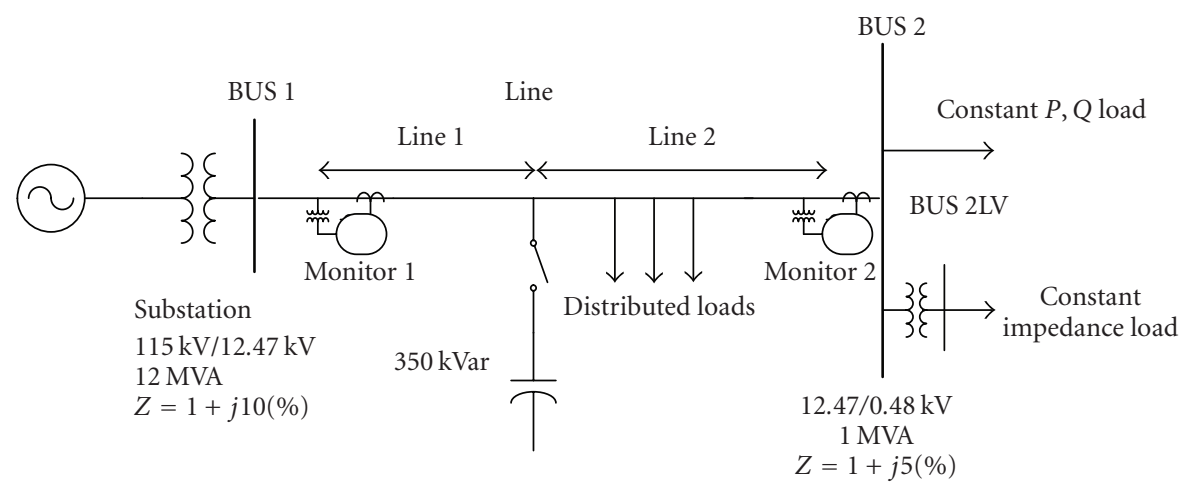

FIgURE 3: IEEE distribution system test case with modification and additional capacitor bank.

Ohms per mile are as follows, respectively:

$$
\begin{aligned}
& {\left[Z_{012}\right]_{U B}} \\
& =\left[\begin{array}{ccc}
0.7737+j 1.9078 & 0.0072-j 0.0100 & -0.0123-j 0.0012 \\
-0.0123-j 0.0012 & 0.3061+j 0.6334 & -0.0488+j 0.0281 \\
0.0072-j 0.0100 & 0.0487+j 0.0283 & 0.3061+j 0.6334
\end{array}\right],
\end{aligned}
$$

$\left[Z_{012}\right]_{B}$

$$
=\left[\begin{array}{ccc}
0.7737+j 1.9078 & 0 & 0 \\
0 & 0.3061+j 0.6333 & 0 \\
0 & 0 & 0.3061+j 0.6334
\end{array}\right] .
$$

The positive sequence line inductance per mile, $L_{u}$, for both balanced and unbalanced feeders is $1.6801 \mathrm{mH} / \mathrm{mi}$. The efficacy of the proposed technique is evaluated under the following conditions: (a) ignore loads and circuits downstream from the switched capacitor bank when all lines are assumed balanced, (b) include loads and circuits downstream from the bank and vary the loading conditions when the loads and lines are assumed balanced, and investigate the feasibility of the proposed method when harmonic currents are injected from the nonlinear loads and resonance occurs as well, and (c) evaluate the same system as in (b), however, loads and lines are unbalanced. Loads illustrated in Figure 3 are modeled as a combination of fixed impedance and dominant complex constant power loads which are appropriately modeled as variable $R$ and $L$ in parallel. They are connected at the $12.47 \mathrm{kV}$ as well as at the $0.48 \mathrm{kV}$ level through a 1 MVA service transformer $Z(\%)=(1+j 5)$. A $350 \mathrm{kVar}$ three phase switched capacitor bank is located $\mathrm{d}_{1}$ miles out on the feeder. Two PQ monitors are installed both at the BUS 1 (substation) and BUS 2. Note that the conventional sampling rate of 256 samples/cycle is applied in the following studies.
TABLE 1: Estimation results for case (a) with $d_{1}=3$ miles.

\begin{tabular}{l|ccc}
\hline Parameters & $f_{\text {res }}=\omega_{d} / 2 \pi$ & $\zeta$ & $X / R$ \\
\hline Analytical results & 707.36 & 0.0139 & 35.96 \\
Estimates & 706.42 & 0.0135 & 37.05 \\
\hline
\end{tabular}

\subsection{Evaluation cases with downstream loads and circuits omitted}

The damping estimation technique is evaluated for a balanced feeder, and loads and circuits downstream from the capacitor bank are excluded from the simulation model. The estimated parameters are compared with the analytical results derived from the characteristic equation in (9) and summarized in Table 1 (for $d_{1}=3$ miles).

The above results show that the proposed techniques provide reasonably accurate estimates of resonant frequency, damping ratio, and effective $X / R$ ratio. Note that the resonant frequency in the resulting table indicates a damped resonant frequency, which is the frequency obtainable from the measurement data. However, the damped resonant frequency is very close to the natural resonant frequency since in general the damping ratio is very small. It should also be noted that the fractional numbers are not included to indicate the high accuracy of the estimates but to present the same significant figures as those of the analytical values. The frequency interval, $\Delta f$, between two closely spaced FFT spectral lines is $15.03 \mathrm{~Hz}$ based on the number of samples (1024) and sampling rate of the PQ data (256 samples per cycle).

\subsection{Evaluation cases for balanced lines and balanced loads}

\subsubsection{Linear load}

In this case, three phase balanced lines and loads downstream from the capacitor bank are included. The lines are configured as $d_{1}=3$ miles and $d_{2}=1$ mile. Note that loads are modeled with series $R$ and $L$ in an aggregate manner and 


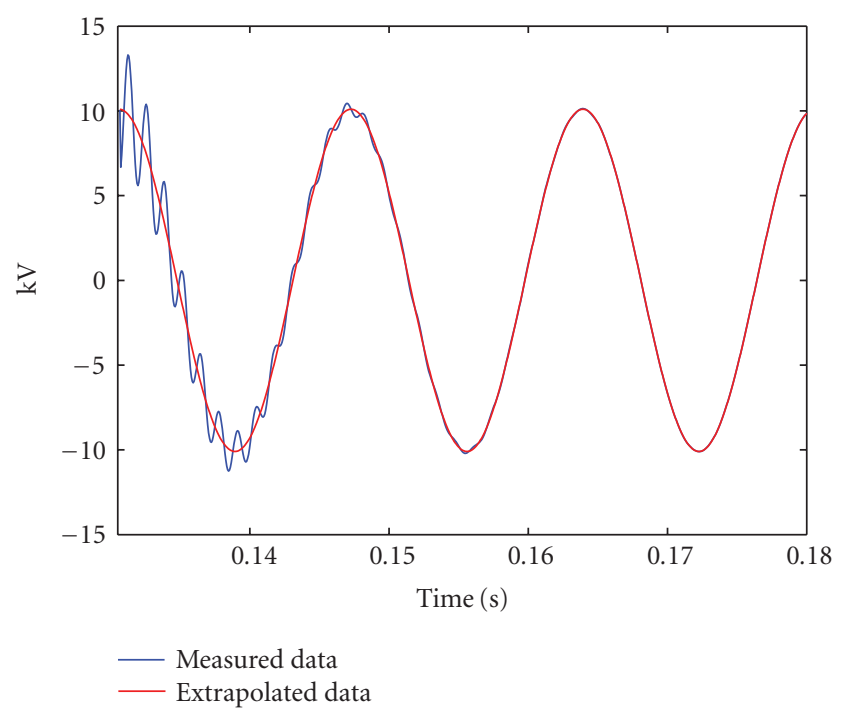

(a)

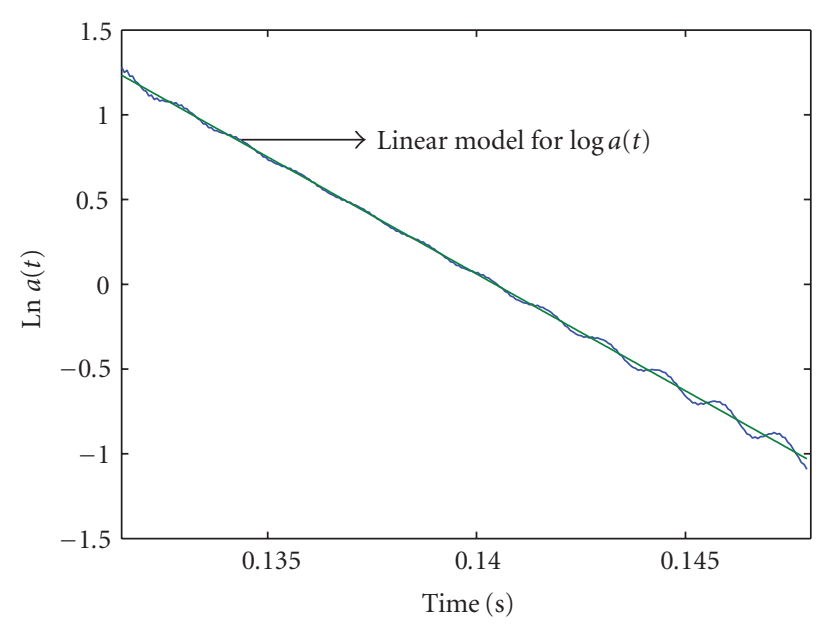

(c)

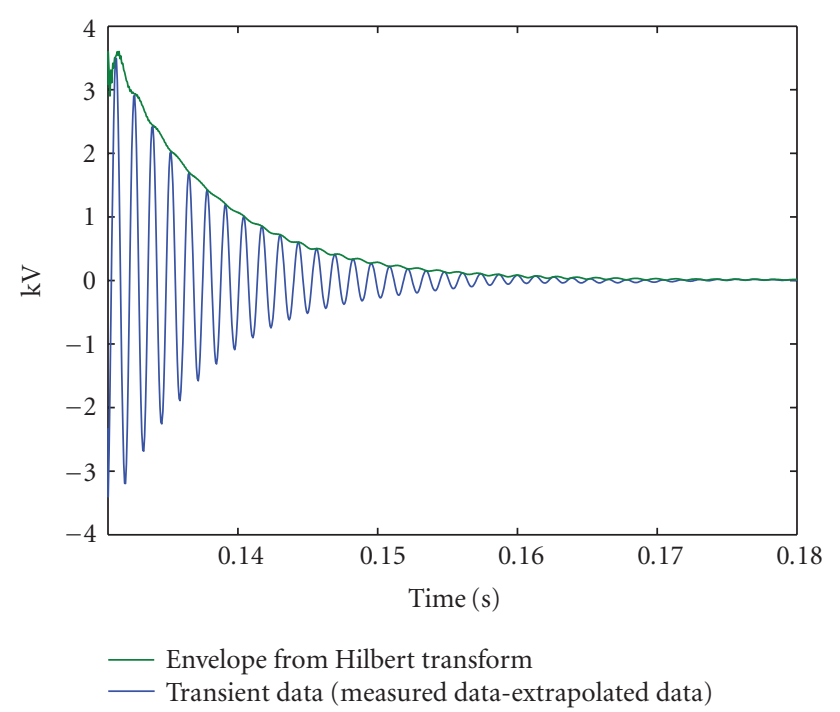

(b)

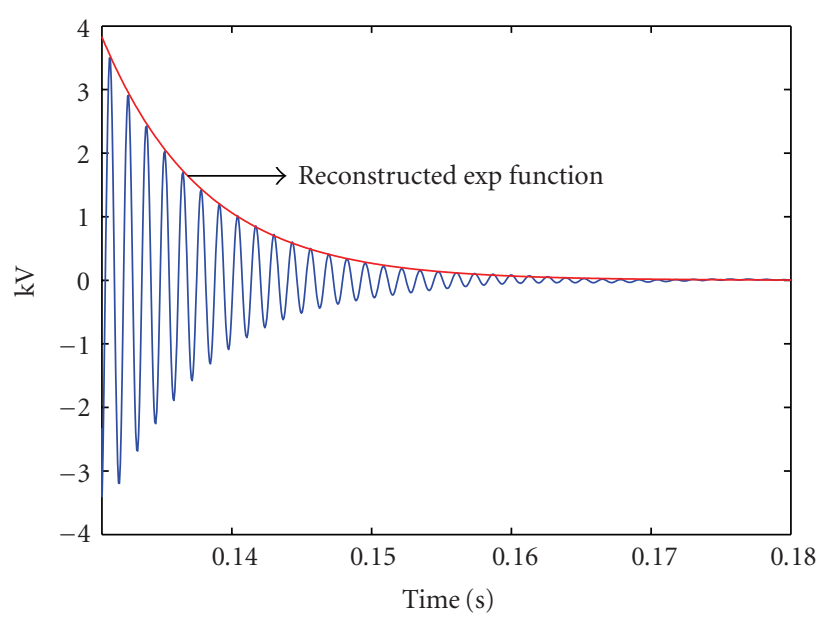

(d)

FIGURE 4: Step-by-step procedures of the proposed damping estimation method. (a) Extracting the transient voltage differential between the measured data (bold) and the extrapolated data (solid), (b) detecting envelope by way of Hilbert transform, (c) performing linear regression for the natural logarithms of the envelope, which results in the effective $X / R$ ratio, and (d) reconstructing exponential function that perfectly fits in the voltage transient response.

TABLE 2: Estimation results when load power factor is 0.95 .

\begin{tabular}{l|ccc|ccc}
\hline $\begin{array}{l}\text { Loading } \\
\text { condition }\end{array}$ & \multicolumn{3}{|c|}{ Moderate, 3.16 MVA } & \multicolumn{3}{|c}{ Heavy, 7.37 MVA } \\
\hline Parameters & $\begin{array}{l}f_{\text {res }}= \\
\omega_{d} / 2 \pi\end{array}$ & $\zeta$ & $X / R$ & $\begin{array}{c}f_{\text {res }}= \\
\omega_{d} / 2 \pi\end{array}$ & $\zeta$ & $X / R$ \\
$\begin{array}{l}\text { Analytical } \\
\text { results }\end{array}$ & 772.28 & 0.0293 & 17.08 & 845.97 & 0.0387 & 12.92 \\
Estimates & 766.55 & 0.0286 & 17.47 & 841.70 & 0.0373 & 13.38 \\
\hline
\end{tabular}

connected to BUS 2. The proposed technique is applied to quantify the system damping level for varying load sizes and power factors. The resulting parameters are compared with
TABLE 3: Estimation results when load power factor is 0.90 .

\begin{tabular}{|c|c|c|c|c|c|c|}
\hline Loading & Mode & ate, 3.16 & MVA & Hea & $y, 7.37 \mathrm{~N}$ & VA \\
\hline Parameters & $\begin{array}{l}f_{\text {res }}= \\
\omega_{d} / 2 \pi\end{array}$ & $\zeta$ & $X / R$ & $\begin{array}{l}f_{\text {res }}= \\
\omega_{d} / 2 \pi\end{array}$ & $\zeta$ & $X / R$ \\
\hline $\begin{array}{l}\text { Analytical } \\
\text { results }\end{array}$ & 758.51 & 0.0217 & 23.00 & 818.11 & 0.0273 & 18.31 \\
\hline Estimates & 751.52 & 0.0214 & 23.38 & 811.64 & 0.0266 & 18.80 \\
\hline
\end{tabular}

the analytical results using the characteristic equation in (18) and summarized in Tables $2-4$. The results demonstrate that the proposed technique can provide very accurate estimates 
TABLE 4: Estimation results when load power factor is 0.87 .

\begin{tabular}{l|ccc|ccc}
\hline $\begin{array}{l}\text { Loading } \\
\text { condition }\end{array}$ & \multicolumn{3}{|c|}{ Moderate, 3.16 MVA } & \multicolumn{3}{|c}{ Heavy, 7.37 MVA } \\
\hline Parameters & $\begin{array}{c}f_{\text {res }}= \\
\omega_{d} / 2 \pi\end{array}$ & $\zeta$ & $X / R$ & $\begin{array}{l}f_{\text {res }}= \\
\omega_{d} / 2 \pi\end{array}$ & $\zeta$ & $X / R$ \\
$\begin{array}{l}\text { Analytical } \\
\text { results }\end{array}$ & 754.58 & 0.0198 & 25.23 & 810.24 & 0.0242 & 20.69 \\
Estimates & 751.52 & 0.0194 & 25.74 & 811.64 & 0.0234 & 21.38 \\
\hline
\end{tabular}

of resonant frequency, damping ratio, and effective $X / R$ ratio. It is also observed that the overall system damping level is more affected by the power factor of the load than the load size. The effective $X / R$ ratio of a moderate load with $0.95 \mathrm{pf}$ is even less than that of heavy load with $0.90 \mathrm{pf}$. Note the change in resonant frequency according to the load condition. The following (21) describes an example of system model reduction process for a moderate loading condition with $0.95 \mathrm{pf}$. The rapid mode truncation reduces the order of transfer function from (10) to (18). The resulting characteristic equation is presented in (22) by taking appropriate numeric values for line parameters according to the positive sequence equivalent circuit;

$$
\begin{gathered}
\frac{V_{S}(s)}{V_{s c}(s)}=\frac{1.278 s^{3}+1.495 e 3 s^{2}+4.778 e 7 s+48.02 e 9}{2.15 s^{3}+2.65 e 3 s^{2}+5.125 e 7 s+48.15 e 9} \\
\Longrightarrow \frac{0.59455\left(s^{2}+132.6 s+3.732 e 7\right)}{s^{2}+284.2 s+2.357 e 7} \\
\Delta(s)=s^{2}+284.2 s+2.357 e 7
\end{gathered}
$$

Note that transient voltage response in any monitoring location in the power system of interest is governed by the same characteristic equation. In fact, the estimates and the theoretical results for the system damping level at PQM 1, 2 and over capacitor location are identical. Figure 4 illustrates the damping estimation procedures. The steps can be summarized as: (a) detecting the capacitor switching time instant; (b) selecting a single cycle of steady state PQ data by extracting a cycle of data after passing one or two cycles from the switching instant, or a single cyclic data right before the capacitor bank energizing when there is insufficient data after the switching event; (c) this extracted single cycle can be concatenated to form a virtual steady-state data based on our assumption that the data is stationary; (d) computing the one cycle difference between the actually measured data and the virtual steady-state data from the switching instant. This results in the empirical-free response of the capacitor bank energizing or the pure transient voltage portion. The damped resonant frequency is accurately determined using the parallel resonant frequency estimation method addressed in [15].

\subsubsection{Nonlinear load}

In this situation, Table 5 presents the estimation results when harmonic currents are injected from the nonlinear loads.

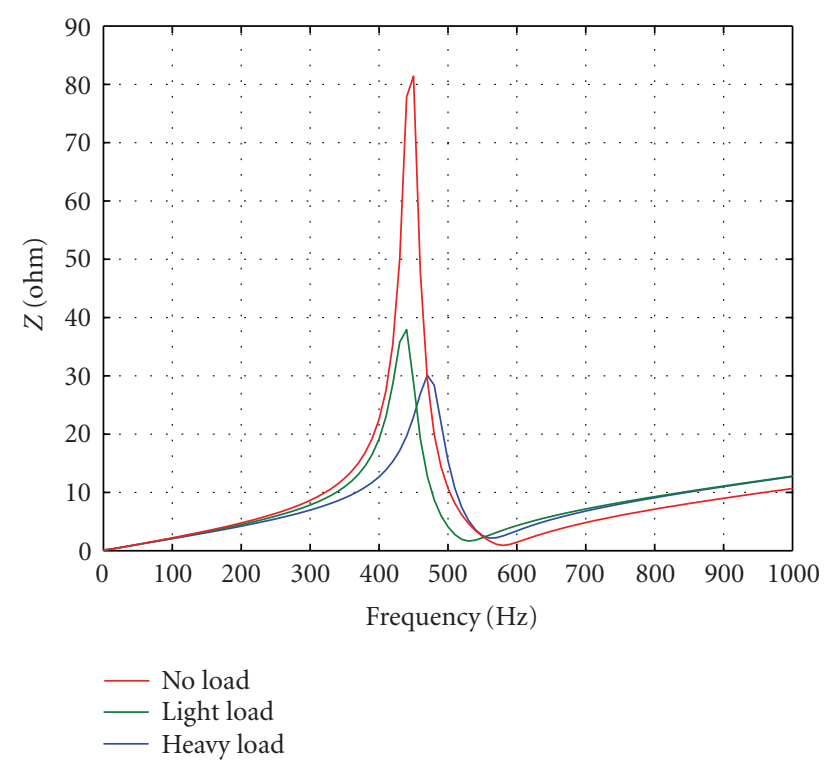

FIGURE 5: System impedance scan results of a typical $12.47 \mathrm{kV}$ system for two different loading conditions.

The load model with power factor of 0.87 is modified to inject the fifth and seventh harmonic currents by $3 \%$ of the $60 \mathrm{~Hz}$ component and the capacitor bank size is increased to $850 \mathrm{kVar}$ to support the resonance condition near the seventh harmonic. The distribution feeder is balanced with $d_{1}=4$ miles and $d_{2}=1$ mile. Both moderate and heavy loading conditions with the same power factor are investigated. The impedance scan results and the voltage and current waveforms are illustrated in Figures 5 and 6 to emphasize the load impact on the system damping and resonant frequency. The change from a heavy to a moderate load condition causes a system resonance phenomenon due to the new resonant frequency formed near at the seventh harmonic as well as the increased peak impedance level. Thus, injecting the same amount of harmonic currents can result in different levels of distorted voltage and current waveforms. However, it is often neglected that change in the load condition shifts the resonant frequency. This can be more influential in mitigating the resonance phenomena in many cases than lowered peak impedance level. The estimation results presented in Table 5 demonstrate that the performance of the proposed technique is independent of the load type, that is, whether it is linear or nonlinear, as long as the steady-state voltage waveforms are considered to be (quasi)-stationary during the observation period immediately after the capacitor bank operation. The estimated parameters are very close to those theoretical values calculated from a positive-sequence equivalent circuit as well.

\subsection{Evaluation case for unbalanced lines and loads}

In this case, the system is modeled with unbalanced lines and loads with $d_{1}=3$ miles and $d_{2}=2$ miles. The resulting 


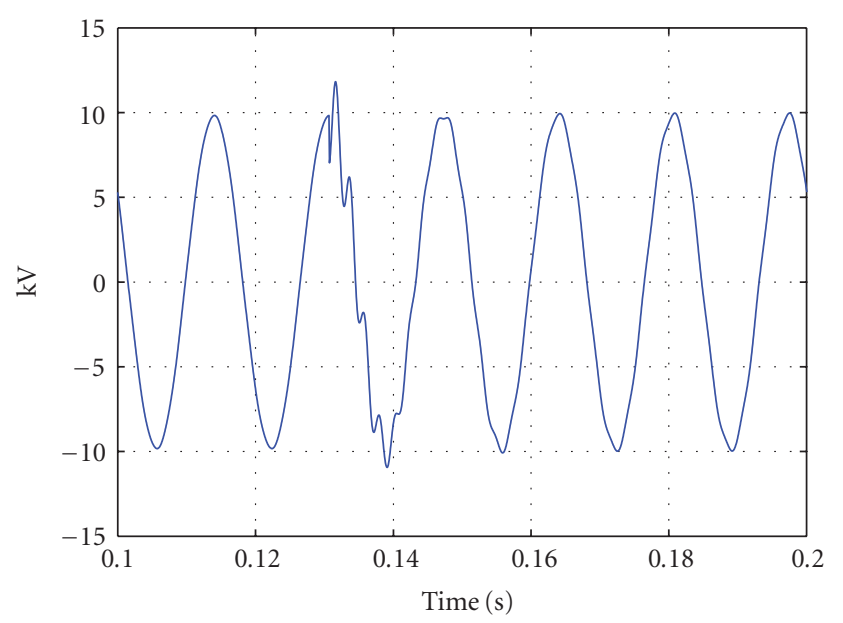

(a)

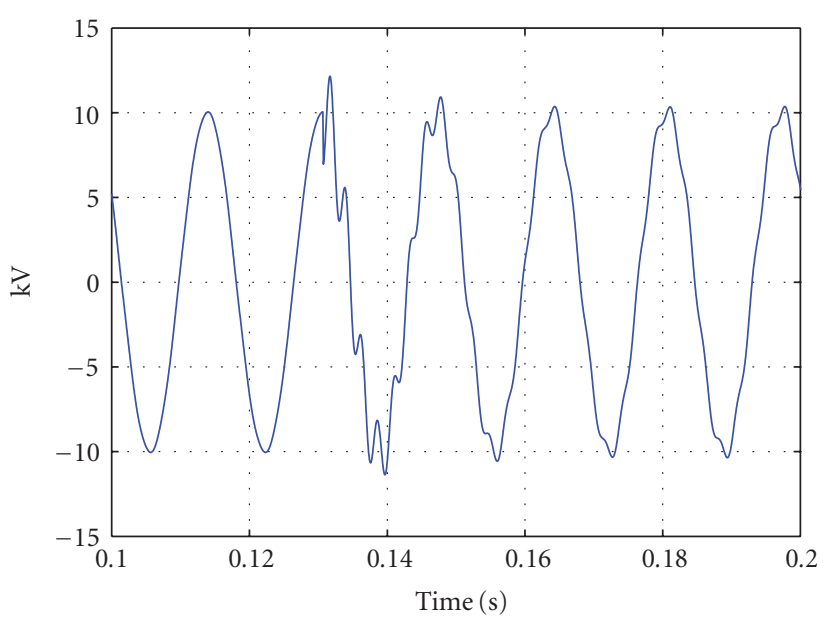

(c)

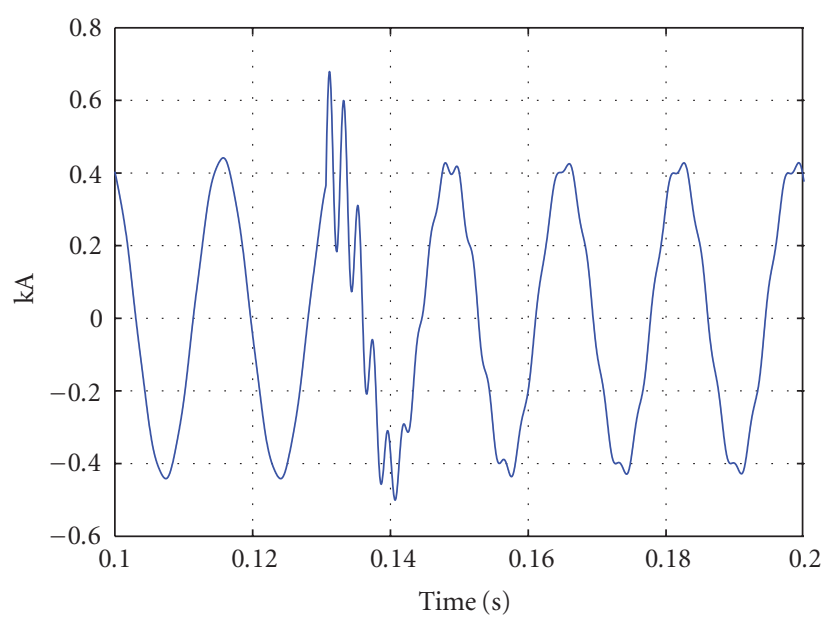

(b)

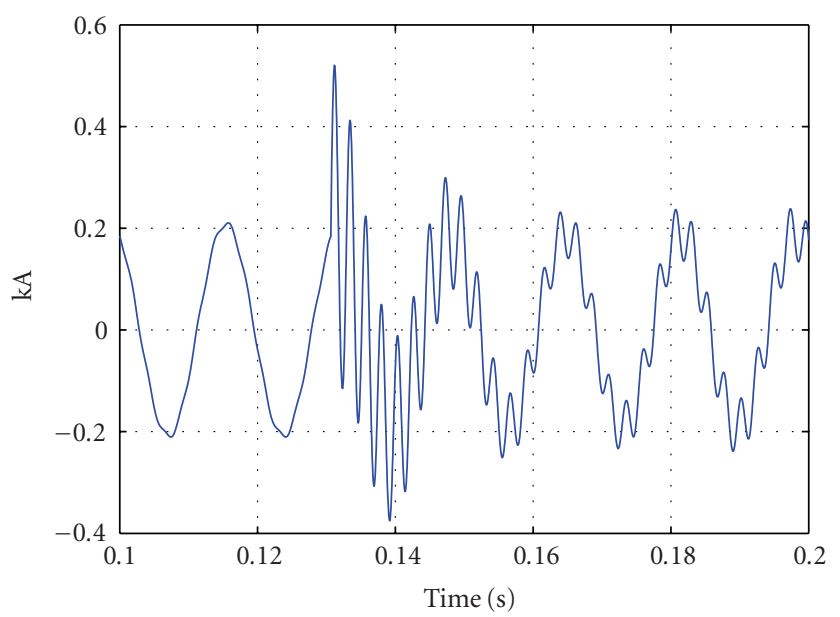

(d)

FIgURE 6: Voltage and current waveforms at a simulated $12.47 \mathrm{kV}$ substation: (a), (b) voltage and current for a system under heavy loading condition and (c), (d) voltage and current when resonance occurs due to loading condition change.

voltage unbalance is $0.5 \%$. Note that only a moderate load size is considered in this case $\mathrm{C}$, since the dominant complex constant power load is modeled by a combination of $R L$ in parallel. The damping from the load then becomes significantly higher compared to that from the combination of $R L$ in series which are employed in the case B. Although the lines and loads are unbalanced, the positive sequence equivalent circuit is analyzed to provide approximate theoretical values using three-phase active and reactive power measured at the substation - 2.91 MVA, 0.92 lagging pf. Although the estimates from each phase show slight deviations, it should be judged that the results are reasonably accurate since they are in the region of expected theoretical values as presented in Table 6. It is also observed that the method is independent of the load composition since only the waveform data is needed. As illustrated in Figure 7, the voltage transient is much shorter than that of the balanced line case. Thus, care must be taken to select the observation period to guarantee the optimal envelope from Hilbert transform: empirical study recommends less than a half-cycle data for this high damping case. Note that the effective $X / R$ ratio is in the order of 1 or 2 . The $X / R$ ratio is approximately $5 \%$ of the isolated capacitor bank case, which has been conventionally employed for harmonic studies. Therefore, thorough understanding of the load type, composition, and condition is required in advance to perform any mitigation measures against harmonic issues and the proposed technique provides system impedance characteristic in a very practical but precise manner.

\section{METHOD APPLICATION USING ACTUAL MEASUREMENT DATA}

The performance of the damping estimation technique is also validated using actual data of a capacitor switching transient event. The transient event was captured using a widely 
TABLE 5: Estimation results for nonlinear load.

\begin{tabular}{l|ccc|ccc}
\hline $\begin{array}{l}\text { Loading } \\
\text { condition }\end{array}$ & \multicolumn{3}{|c|}{ Moderate, 3.16 MVA } & \multicolumn{3}{|c}{ Heavy, 7.37 MVA } \\
\hline Parameters & $\begin{array}{c}f_{\text {res }}= \\
\omega_{d} / 2 \pi\end{array}$ & $\zeta$ & $X / R$ & $\begin{array}{c}f_{\text {res }}= \\
\omega_{d} / 2 \pi\end{array}$ & $\zeta$ & $X / R$ \\
$\begin{array}{l}\text { Analytical } \\
\text { results }\end{array}$ & 439.58 & 0.0374 & 13.38 & 476.94 & 0.0454 & 11.02 \\
Estimates & 439.27 & 0.0370 & 13.50 & 476.45 & 0.0437 & 11.43 \\
\hline
\end{tabular}

TABLE 6: Estimation results with unbalanced lines and loads.

\begin{tabular}{c|cccc}
\hline & Phase A & Phase B & Phase C & $\begin{array}{l}\text { Theoretical } \\
\text { value }\end{array}$ \\
\hline$f_{\text {res }}=\omega_{d} / 2 \pi$ & 766.55 & 766.55 & 766.55 & 762.30 \\
$\zeta$ & 0.2918 & 0.2097 & 0.3732 & 0.3459 \\
$X / R$ & 1.713 & 2.356 & 1.340 & 1.446 \\
\hline
\end{tabular}

available power quality monitoring device at a $115 \mathrm{kV}$ substation of a utility company. Figure 8 illustrates the measured voltage waveforms and the results from the Hilbert damping analysis while Table 7 summarizes the resulting estimated parameters. As shown in Figure 8(d), there are two prominent frequency components at $526 \mathrm{~Hz}$ and $721 \mathrm{~Hz}$. However, the lower component at $526 \mathrm{~Hz}$ is selected to estimate the effective $X / R$ ratio since the magnitude at $526 \mathrm{~Hz}$ is much bigger. Although there are no theoretical values to evaluate the estimation results, the obtained values are considered to be reasonable in that the system is at a subtransmission level whose $X / R$ ratio is generally known to be in the order of 30 , and the envelope nicely matches the transient voltage as shown in Figure 8(c).

\section{DISCUSSIONS}

As indicated in the application to the real data, however, the Hilbert damping analysis may cause considerable estimation errors for the following possible two scenarios: (1) the PQ data is significantly corrupted by noises such that the stationarity assumption on the PQ data is no longer valid; (2) the extracted free response possess multiple comparable resonant frequency components such that there is no single dominant mode. One may consider the following ways around these problems.

(i) Reinforce the signal preprocessing stages by adding the high frequency noise rejection filters and adding the bandpass filters. Thus, one can appropriately select important resonant frequencies based on the system studies followed by the Hilbert damping analysis.

(ii) Exploit the wavelet transform which inherently embeds the bandpass filtering which can provide a unified algorithm to estimate the damping ratios of those multiple

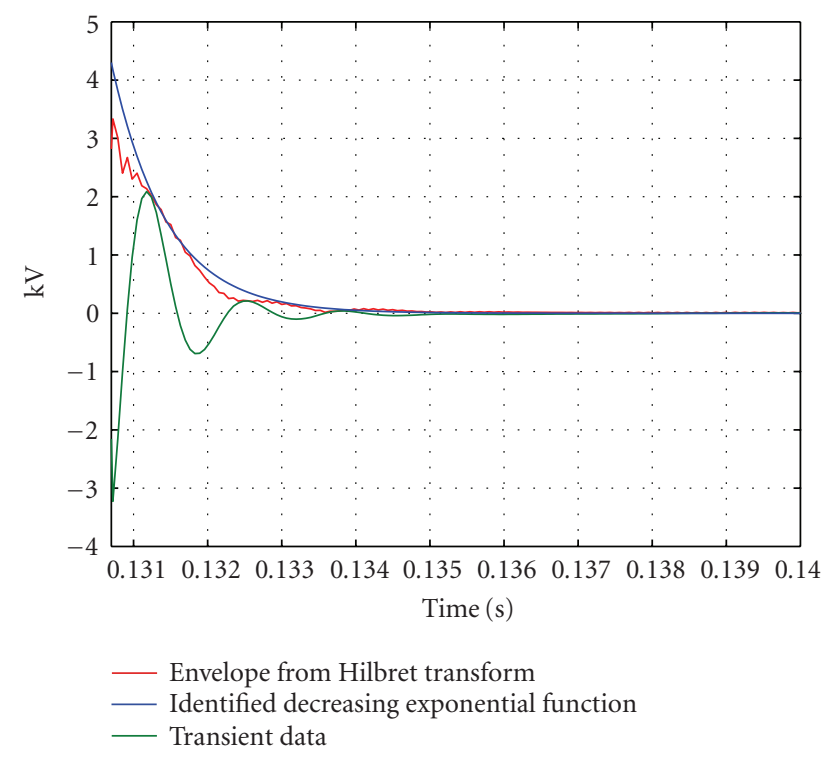

FIGURE 7: Hilbert damping analysis of phase A transient voltage of a moderately loaded system.

TABLE 7: Estimation results for actual data.

\begin{tabular}{c|c}
\hline Parameters & Estimates \\
\hline$f_{\text {res }}=\omega_{d} / 2 \pi$ & 526 \\
$\zeta$ & 0.0154 \\
$X / R$ & 32.50 \\
\hline
\end{tabular}

modes. We will provide this wavelet-based power system damping estimation algorithm in the near future.

(iii) Apply methodology known to be robust to ambient noise signals such as ESPRIT which includes the noise term in its original mathematical model. Thus, one can even extract important system information even from the heavily distorted data at the cost of increased computational burden [7].

\section{CONCLUSIONS}

This paper proposed a novel method to estimate utility distribution system damping. The proposed method is derived using linear dynamic system theory and utilizes the Hilbert system damping analysis to extract circuit signatures describing the system damping embedded in the voltage waveforms. The efficacy of the integrated signal processing and system theory was demonstrated using data obtained from simulations of a representative utility distribution system and an actual power system. The results show that the proposed method can accurately predict the utility distribution system damping parameters. Limitations of the proposed method are discussed with possible solutions suggested. 


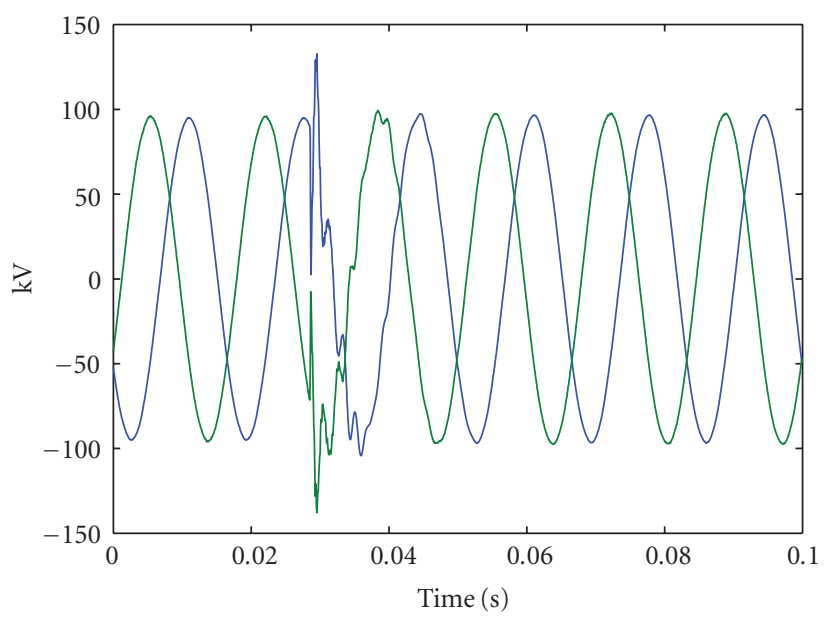

(a)

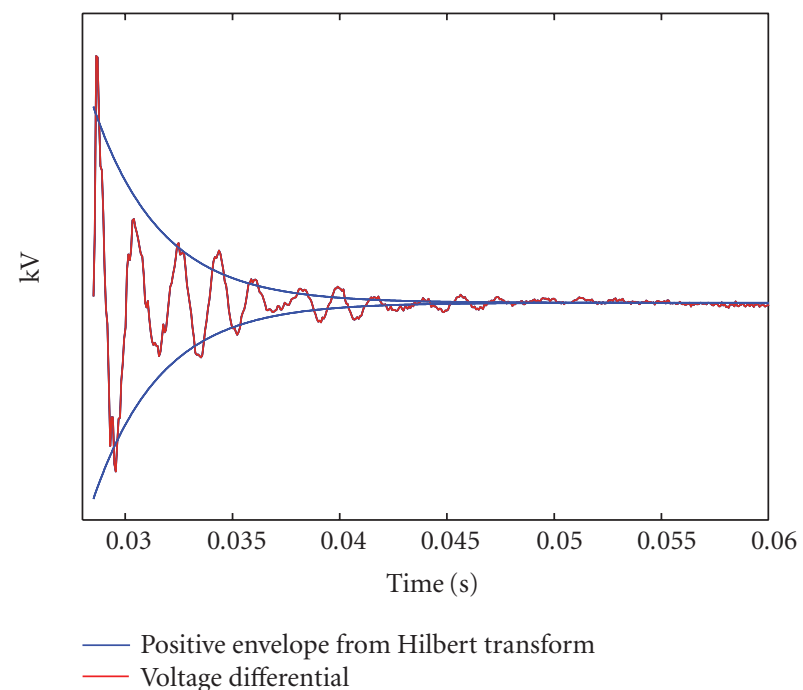

(c)

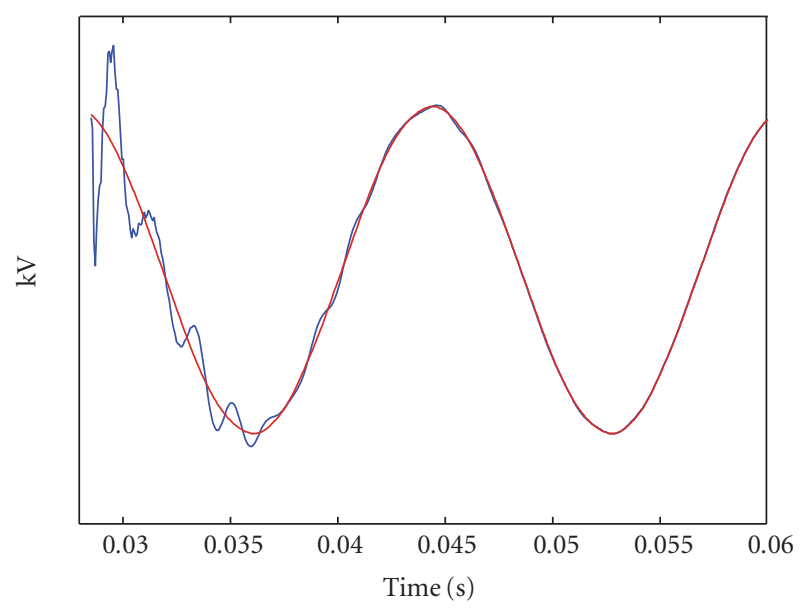

$-\mathrm{Va}$

— Extrapolated data

(b)

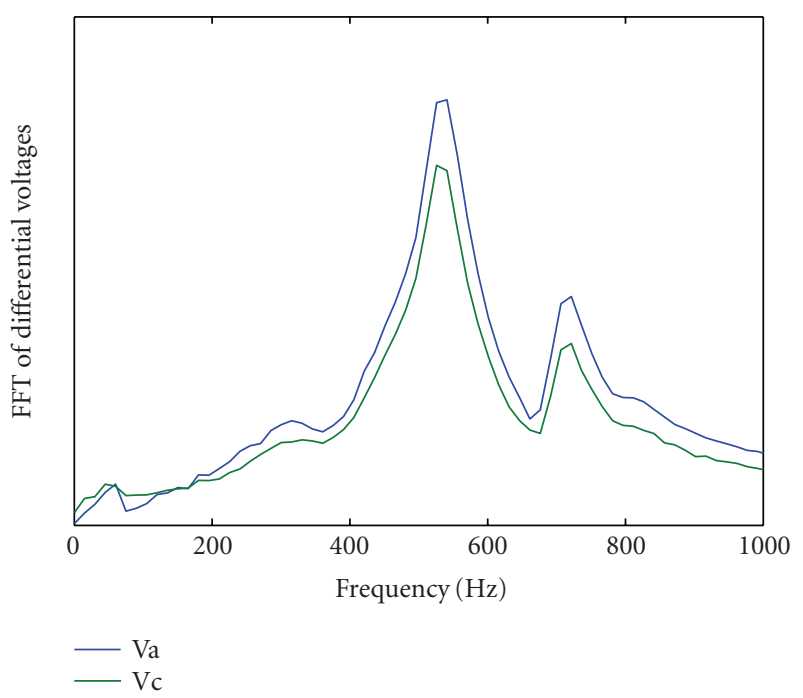

(d)

Figure 8: Application of the damping estimation method to actual data: (a) voltage waveform of phase A (bold) and C, (b) Phase A voltage transient and the extrapolated voltage after capacitor switching, (c) positive and negative envelope of voltage A detected by Hilbert damping analysis, (d) spectral information of the differential voltage A and C.

\section{REFERENCES}

[1] R. D. Dugan, M. F. McGranaghan, S. Santoso, and W. H. Beaty, Electrical Power Systems Quality, McGraw-Hill, New York, NY, USA, 2nd edition, 2003.

[2] T. E. Grebe, "Application of distribution system capacitor banks and their impact on power quality," IEEE Transactions on Industry Applications, vol. 32, no. 3, pp. 714-719, 1996.

[3] M. Banejad and G. Ledwich, "Quantification of damping contribution from loads," IEE Proceedings: Generation, Transmission and Distribution, vol. 152, no. 3, pp. 429-434, 2005.

[4] J. F. Hauer, "Application of Prony analysis to the determination of modal content and equivalent models for measured power system response," IEEE Transactions on Power Systems, vol. 6, no. 3, pp. 1062-1068, 1991.

[5] P. Kundur, Power System Stability and Control, EPRI, Palo Alto, Calif, USA, 1994.

[6] M. H. J. Bollen, E. Styvaktakis, and I. Y. H. Gu, "Categorization and analysis of power system transients," IEEE Transactions on Power Delivery, vol. 20, no. 3, pp. 2298-2306, 2005.

[7] I. Y. H. Gu and E. Styvaktakis, "Bridge the gap: signal processing for power quality applications," Electric Power Systems Research, vol. 66, no. 1, pp. 83-96, 2003.

[8] PES Distribution Systems Analysis Subcommittee, Radial Test Feeders. IEEE, http://ewh.ieee.org/soc/pes/dsacom/testfeeders. html. 
[9] Manitoba HVDC Research Centre, Winnipeg, Canada. PSCAD/EMTDC version 4.2.

[10] J. S. Bendat and A. G. Piersol, Random Data: Analysis and Measurement Procedures, John Wiley \& Sons, New York, NY, USA, 1986.

[11] B. C. Kuo and F. Golnaraghi, Automatic Control Systems, John Wiley \& Sons, New York, NY, USA, 8th edition, 2003.

[12] A. Greenwood, Electrical Transients in Power Systems, John Wiley \& Sons, New York, NY, USA, 2nd edition, 1991.

[13] A. C. Antoulas, Approximation of Large-Scale Dynamical Systems, SIAM, Philadelphia, Pa, USA, 2005.

[14] S. Santoso, E. J. Powers, W. M. Grady, and P. Hofmann, "Power quality assessment via wavelet transform analysis," IEEE Transactions on Power Delivery, vol. 11, no. 2, pp. 924 930, 1996.

[15] K. Hur and S. Santoso, "An improved method to estimate empirical system parallel resonant frequencies using capacitor switching transient data," IEEE Transactions on Power Delivery, vol. 21, no. 3, pp. 1751-1753, 2006.

[16] L. L. Scharf, Statistical Signal Processing: Detection, Estimation and Time-Series Analysis, Addison Wesley, New York, NY, USA, 1991.

Kyeon Hur received his B.S. and M.S. degrees in electrical engineering from Yonsei University, Seoul, Korea, in 1996 and 1998. He was with Samsung Electronics as an R\&D Engineer between 1998 and 2003, where he designed control algorithms for electric drives. He is now a Ph.D candidate in electrical and computer engineering at The University of Texas at Austin. His areas of interest include power quality, power electronics, renewable energy, and the application of novel digital signal processing techniques to nonlinear and/or transient problems in engineering. He is a recipient of KOSEF (Korea Science and Engineering Foundation) Graduate Scholarship.

Surya Santoso has been an Assistant Professor with Department of Electrical and Computer Engineering, The University of Texas at Austin since 2003. He was a Senior Power Systems/Consulting Engineer with Electrotek Concepts, Knoxville, TN between 1997 and 2003. He holds the BSEE degree from Satya Wacana Christian University, Indonesia, and the MSEE and Ph.D degrees from the University of Texas at

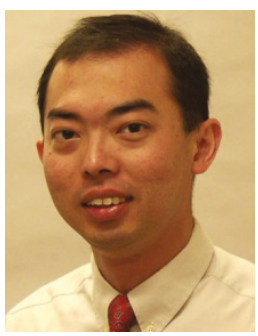
Austin. His research interests include power system analysis, modeling, and simulation. He is a Coauthor of Electrical Power Systems Quality published by McGraw-Hill, now in its 2 nd edition. He chairs a task force on intelligent system applications to data mining and data analysis, and a Member of the IEEE PES Power Systems Analysis, Computing and Economics Committee.

Irene Y. H. Gu is a Professor in signal processing at the Department of Signals and Systems at Chalmers University of Technology, Sweden. She received the Ph.D. degree in electrical engineering from Eindhoven University of Technology (NL), in 1992. She was a Research Fellow at Philips Research Institute IPO (NL) and Staffordshire University (UK), and a Lecturer at The

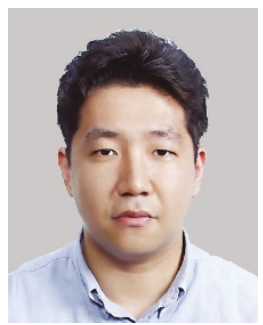

University of Birmingham (UK) during 1992-1996. Since 1996, she has been with Chalmers University of Technology (Sweden). Her current research interests include signal processing methods with applications to power disturbance data analysis, signal and image processing, pattern classification and machine learning. She served as an Associate Editor for the IEEE Transactions on Systems, Man and Cybernetics during 2000-2005, the Chair-Elect of Signal Processing Chapter in IEEE Swedish Section 2002-2004, and is a Member of the Editorial Board for Applied Signal Processing since July 2005. She is the Coauthor of Signal processing of power quality disturbances published by Wiley/IEEE-Press 2006.

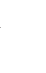

